\title{
Cooperative ramp metering simulation
}

\author{
Martin Gregurić, Edouard Ivanjko and Sadko Mandžuka \\ University of Zagreb Faculty of Transport and Traffic Sciences/Department of Intelligent Transportation Systems, \\ Zagreb, Croatia

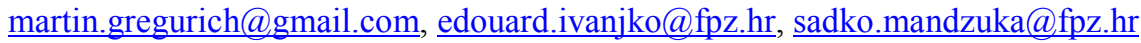

\begin{abstract}
The increase of vehicle numbers in recent decades resulted in road traffic congestion problems. Such congestions are a characteristic of densely populated urban areas and occur daily during morning and afternoon rush hours. Urban areas have been suffering from the lack of space needed to build new road infrastructure. The traffic congestion problem can be solved by applying new traffic control approaches from the domain of intelligent transportation systems (ITS). One of the applied methods from ITS is known as ramp metering and is used to increase the throughput of urban highways with many on- and offramps. Nowadays ramp metering is used in cooperation with additional control approaches like variable speed limit control (VSLC). Prior to implementation, such cooperative traffic control systems have to be tested in simulations using real world traffic data. One of the used simulators is CTMSIM which enables macroscopic simulation of highway traffic and local ramp metering approaches. In this paper the CTMSIM simulator is augmented to enable simulation of cooperative ramp metering algorithms, stand-alone VSLC, and cooperation between ramp metering and VSLC. Augmented simulator is tested using some limited available traffic data with the Zagreb bypass urban highway as a case study.
\end{abstract}

\section{INTRODUCTION}

Urban highways cannot fulfil originally projected high level of service (LoS) today anymore. LoS is defined as a group of qualitative measures which characterize operational conditions within traffic flow and their perception by motorists and drivers [1]. Main reason for low values of LoS on today's urban highways is high amount of traffic during increased daily (also known as rush hour) or seasonal traffic demand. In the same time urban highways are in most cases integrated into the urban road network and environment so there is no more space for infrastructural build-up.

On- and off-ramps on urban highways are common places for bottlenecks since they are the merging points of mainstream and on-ramp flow. Congestions at on-ramps become problematic if mainstream and/or on-ramps are overburdened with heavy transit traffic or/and a large number of local drivers. Local drivers prefer to use the urban highway instead of the local urban road network in time of daily migration [2]. Such a traffic scenario is common at most urban highways.

Regarding mentioned problems, new traffic control concepts are being applied to ensure desired LoS. On- ramp merging control method known as ramp metering and mainstream variable speed limit control (VSLC) are the most used control methods on urban highways [3]. Ramp metering and VSLC are important services used in intelligent transportation system (ITS) based traffic control architectures.

Ramp metering implementations are generally simulated by using various traffic simulation programs with the ability to simulate interaction of all existing traffic flows in a highway system. Traffic simulators are divided into two major categories: macroscopic and microscopic. Macroscopic simulators compute cumulative traffic flow parameters (e.g. speed, flow, and density) and their relationships to each other according to traffic flow equations. Individual vehicles are not considered. Most used macroscopic simulators are: CTMSIM, FREFLO, AUTOS, METANET and VISUM. Microscopic simulators continuously or discretely compute parameters (e.g. position, speed, maximum acceleration rate, etc.) of every individual vehicle during simulation. Most used microscopic simulators are: PARAMICS, MITSIM, CORSIM, VISSIM, AIMSUN, and TRANSIM.

This paper tackles the problem of cooperative highway traffic control systems simulation using a macrosimulation environment. CTMSIM is selected as a suitable macroscopic-highway simulator because of its open architecture based on Matlab. New functionalities and control approaches can be easily added and verified. In order to perform analysis and to validate the impact of cooperative control systems on highway traffic flows, it is necessary to adequately extend CTMSIM. In its original version it only supports local ramp metering algorithms without the possibility to simulate cooperative control approaches between several on-ramps or speed limit control (SLC) systems.

CTMSIM simulator is augmented to support SLC using a modified graphical user interface (GUI) to set a fixed speed limit value for every segment (cell) of the highway model. This value is used as the default speed limit value. Additionally, it is possible to change the speed limit value during simulation, which transforms SLC into variable SLC (VSLC). In order to enable cooperative ramp metering simulation, a hierarchical control simulation framework is developed and added to 
CTMSIM. Using mentioned augmentation cooperative ramp metering algorithm, HELPER is implemented to verify the new framework. Augmented CTMSIM also enables simulation of cooperation between the implemented ramp metering algorithm HELPER and VSLC. Zagreb bypass highway is used as test case for evaluation of the developed hierarchical control framework and VSLC.

This paper is organized as follows. Second section briefly describes concept and problems which can be mitigated by application of ramp metering. Concept of cooperation between on-ramps and other highway control systems in order to achieve better results in ramp metering is presented in third section. Fourth and fifth sections describe basic features of the CTMSIM simulation environment and implemented augmentations. Sixth section contains simulation results of the comparative analysis of implemented ramp metering algorithms, VSLC and cooperative approaches. Paper ends with conclusion and future work description.

\section{RAMP METERING}

Common source of congestions on urban highways is merging of mainstream and on-ramp traffic flows as presented in Fig. 1. Area where these two types of traffic flows are actually interacting is known as downstream bottleneck. Consequences of highway congestions manifest through the following indicators: traffic demand exceeding road capacity, increased number of accidents and incidents, on-ramps queues that spill over into the urban arterial roads and peaks in traffic demand resulting from platooned vehicle entries from on-ramps [4]. Location of the downstream bottleneck close to the onramp and the spillback effect on the adjacent local urban road network is denoted in Fig. 1.

The main objective of ramp metering is to control queues at on-ramps in order to maintain maximal capacity flow on a highway. This is achieved by restricting access of on-ramp traffic to mainstream traffic with special traffic lights and appropriate signalization [5]. Visualization of complete installation of a local ramp metering system on a highway is shown in Fig. 2. Most important part of the ramp metering system is the algorithm which determines the "access rate reduction" for every on-ramp flow.

Generally it is possible to divide ramp metering algorithms in two major categories: local (or isolated) and coordinated. Local strategies take into account only traffic conditions on particular on-ramp and nearby segment of

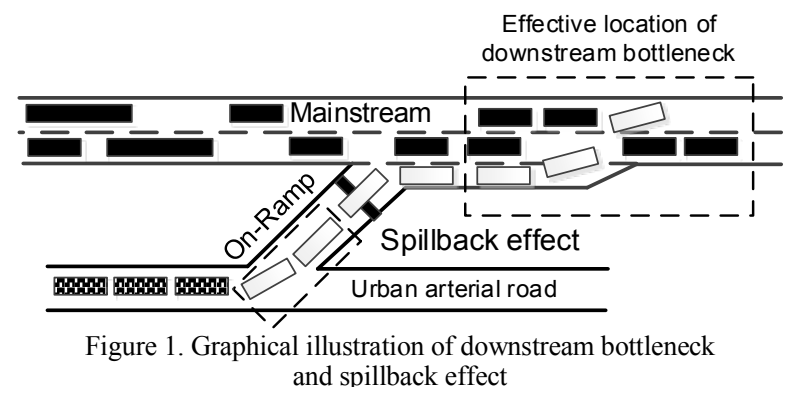

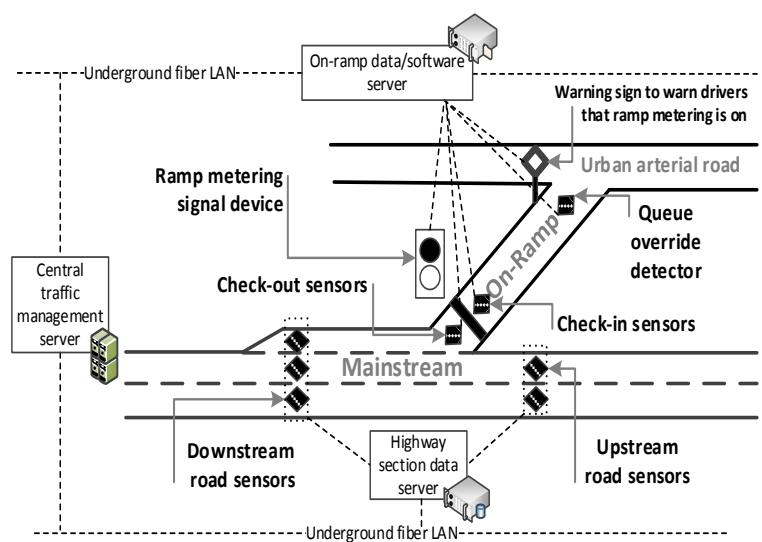

Figure 2. Basic ramp metering installation on a highway section

highway where they are applied. Most representative member of this algorithm category is ALINEA. Main working principle of ALINEA is to keep the downstream occupancy of the on-ramp at a predefined value using a closed loop control structure [6].

Coordinated algorithms consider traffic situation on the overall highway system. According to overall highway traffic state, metering rate for every on-ramp is adjusted. These coordinated ramp metering algorithms are further divided into cooperative, competitive and integrated algorithms. Simulation of cooperative algorithms is addressed in this paper and therefore they will be explained in the next section with more details. Competitive algorithms first compute two or more sets of metering rates using different algorithms types. The more restrictive one will be selected as the actually implemented rate. Typical representative of competitive ramp metering algorithms is SWARM. The SWARM algorithm has two major modules. First module performs local, while second module conducts global predictive ramp metering based on the same traffic control scenario. Metering rates for every on-ramp obtained by local and global algorithm are compared and smaller value is selected as final ramp metering rate [7]. Integrated algorithms are based on optimization of a specific LoS value while considering constraints such as maximum allowable on-ramp queue, bottleneck capacity, etc.

Some authors also make an alternative classification of real-time (traffic-responsive) and fixed-time ramp metering strategies [6]. Real-time ramp metering strategies are based on real-time traffic data obtained from highway sensors while fixed-time strategies use historical data without insight into the current traffic situation.

\section{COOPERATIVE RAMP METERING}

A cooperative control system is defined as a set of control entities that share information and/or tasks to accomplish a common, though perhaps not singular, objective. Cooperation in ramp metering was originally achieved by exploiting adjacent on-ramps queue capacity in order to perform effective mitigation of congestion related to a particular area with several close on-ramps. In such a highway network configuration, there exists the risk that mainstream congestion originating from an onramp can cause severe congestion that includes one or 
more upstream on-ramps. Such congestion spread closes the access of mainstream vehicles to upstream off-ramps enlarging the congestion problem.

Latest work in cooperative ramp metering includes cooperation between classic cooperative ramp metering framework and other highway management services, driver information systems and vehicle itself [7]. In such a way, cooperation with additional highway traffic control systems can be established.

\section{A. Cooperation between on-ramps}

As mentioned, main problem with local or isolated ramp metering is a risk of fast upstream congestion propagation known as a "shock wave". This traffic scenario is common at a highway section with an onramp when local ramp metering cannot efficiently control the merging process between high traffic demands at onramp or/and in mainstream. This situation creates a mainstream "shock wave" which in relation to the motion of the vehicles travels backward or upstream. Upstream on-ramps with local ramp metering applied are not aware of downstream traffic conditions and therefore cannot provide an adequate solution to prevent the congestion spread.

Cooperative ramp metering algorithms work in two phases. In the first phase metering rate for each on-ramp is computed by its local algorithm. The second phase involves further adjustment of each local metering rate according to overall highway system information. Overall information involves traffic data from every on-ramp and its associated mainstream section traffic data. This phase is done in a cooperative unit. Main goal of mentioned cooperation is to achieve higher highway throughput by adjusting on-ramp metering rates upstream of the onramp affected by congestion.

HELPER was the first algorithm which used the mentioned cooperative ramp metering principle. It contains local traffic responsive metering algorithm and a cooperative unit. The cooperative unit uses data obtained from local ramp metering units to monitor the whole controlled highway section. If congestion or a long onramp queue is detected, it declares the belonging highway section with its on-ramp as master. Furthermore, several upstream on-ramps are declared as slave on-ramps. Slave on-ramps have the role to create virtual queues in order to reduce queue length on the congested on-ramp.

\section{B. Cooperation with additional traffic control systems}

Except cooperation between several on-ramps, additional cooperation can be established within the form of a superstructure containing several traffic control subsystems. Such sub-systems are: VSLC, line prohibiting changes and various driver information services. In cooperation with the core ramp metering system they can provide better control over involved traffic flows. In this paper, emphasis is on cooperation between VSLC and ramp metering. VSLC is applied shortly before HELPER creates virtual on-ramp queues and ends when the congestion is reduced.

\section{SIMULATOR CTMSIM}

CTMSIM is an interactive simulator based on macroscopic traffic models specifically designed for highway traffic flows simulations. Its original version supports several local and competitive ramp metering algorithms including on-ramp queue control. Highway model is based on the Asymmetric Cell Transmission Model (ACTM) and allows implementation and development of user-pluggable on-ramp flow and queue controllers [8]. In order to apply ACTM, the highway is divided into $I$ cells. Basic ACTM architecture with marked variables is shown in Fig. 3.

Detailed ACTM used in CTMSIM is explained in [8] and in continuation basic equations are given. Simulation time is divided into $K$ intervals with length $\Delta t$. Number of vehicles moving from cell $i$ to cell $i+1$ (or mainstream flow) during interval $k$ can be obtained according to (1):

$$
\begin{gathered}
f_{i[k]}=\min \left\{v_{i}\left(1-\beta_{i[k]}\right)\left(n_{i[k]}+\gamma r_{i[k]}\right),\right. \\
\left.w_{i+1}\left(\bar{n}_{i+1}-n_{i+1}-\gamma r_{i+1[k]}\right), F_{i[k]}\right\}, \\
F_{i[k]} \triangleq \min \left\{\bar{f}_{l}, \frac{1-\beta_{i[k]}}{\beta_{i[k]}} \bar{s}_{i}\right\},
\end{gathered}
$$

where $\beta_{i[k]}$ denotes split ratio for off-ramp flow, and $\gamma$ represents on-ramp flow blending coefficient, both from interval $[0,1]$. The blending coefficients define amount of traffic flow added or separated from mainstream traffic flow before its value is computed [9]. Furthermore, $v_{i}$ denotes normalized free flow speed and $n_{i[k]}$ represents number of vehicles (or mainstream density) in cell $i$ at time step $k \Delta t$. The parameter $r_{i[k]}$ denotes number of vehicle entering cell $i$, from its associated on-ramp at time step $k \Delta t$, while $w_{i+1}$ represents normalized congestion speed in cell $i+1$. $F_{i[k]}$ denotes congested flow which leaves cell $i$, and $\bar{f}_{l}$ represents mainline capacity of cell $i$ while $\bar{s}_{i}$ denotes off-ramp capacity in cell $i$. On-ramp flow values are now determined with constraints given by:

$$
\begin{gathered}
r_{i[k]} \leq l_{i[k]}+d_{i[k]}, \\
r_{i[k]} \leq \vartheta_{i}\left(\bar{n}_{i}-n_{i[k]}\right), \\
r_{i[k]} \leq c_{i[k]},
\end{gathered}
$$

where $l_{i[k]}$, denotes number of vehicles queuing in the onramp in cell $i$ at time $k \Delta t$, and $d_{i[k]}$ represents demand for on-ramp in cell $i$. The parameter $c_{i[k]}$ denotes value of metering rate computed by the chosen ramp metering algorithm, while $\vartheta_{i}$ denotes on-ramp allocator parameter. On-ramp flow (metering rate) that can be merged with mainstream is obtained by:

$$
r_{i[k]}=\min \left\{l_{i[k]}+d_{i[k]}, \vartheta_{i}\left(\bar{n}_{i}-n_{i[k]}\right), c_{i[k]}\right\} .
$$

Equation (7) describes computation of off-ramp flow in cell $i$ during time interval $k$ :

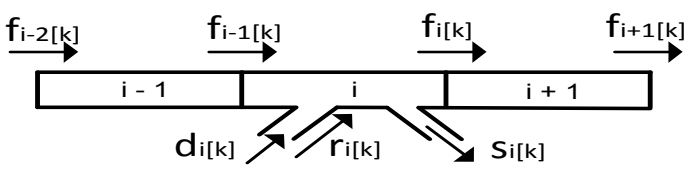

Figure 3. Basic ACTM architecture [9] 


$$
s_{i[k]}=\frac{\beta_{i[k]}}{1-\beta_{i[k]}} f_{i[k]} .
$$

Mainstream density in the cell $i$ during interval $k+1$ can be computed from mainstream conservation equation:

$$
n_{i[k+1]}=n_{i[k]}+f_{i-1[k]}+r_{i[k]}-f_{i[k]}+s_{i[k]} .
$$

Mainstream speed in the cell $i$ is obtained according to the following equation:

$$
v_{i}^{c}=\min \left(\frac{f_{i[k]} /\left(1-\beta_{i[k]}\right)}{n_{i[k]}+\gamma r_{i[k]}}\left(\frac{L_{i}}{\Delta t}\right), v_{i}^{f f}\right),
$$

where $v_{i}^{f f}$ represents free flow speed value for cell $i$, and $L_{i}$ denotes length of cell $i$.

\section{AUGMENTATION OF CTMSIM}

In order to simulate cooperative ramp metering control, additional high level traffic control framework was developed as augmentation of the original CTMSIM simulator as presented in Fig. 4. The ACTM equations are modified to enable definition of a speed limit value for each cell separately.

\section{A. Cooperative ramp metering module}

In order to simulate cooperative highway control systems first step in CTMSIM augmentation was to enable cooperation in ramp metering. This is enabled through the cooperative ramp metering module. As it can be seen in the bottom part of Fig. 4 the original CTMSIM simulation sequence goes only through defined cells in a particular time step. In the proposed augmentation, additional simulation step is added at end of each time step. It is computed after all cells have been simulated. This additional simulation step is related to the cooperative ramp metering module and it has access to data from all cells so it can compute optimal local ramp metering rates and VSLC values used in the next time interval $k$.

First task of this cooperative ramp metering module is to monitor traffic conditions at every on-ramp during simulation. If critical density is exceeded in a current simulation time step, the central monitor module marks this cell's on-ramp as the "master" on-ramp. Metering rate at the "master" on-ramp is then decreased by $10 \%$ of current traffic demand. Three upstream cells with onramps of the "master" on-ramp are declared as "slave" onramps. Metering rate at the "slave" on-ramps are decreased by $40 \%$ of current traffic demand what creates virtual queues on them.

Second task of cooperative module is to enable override possibility for every metering rate, previously

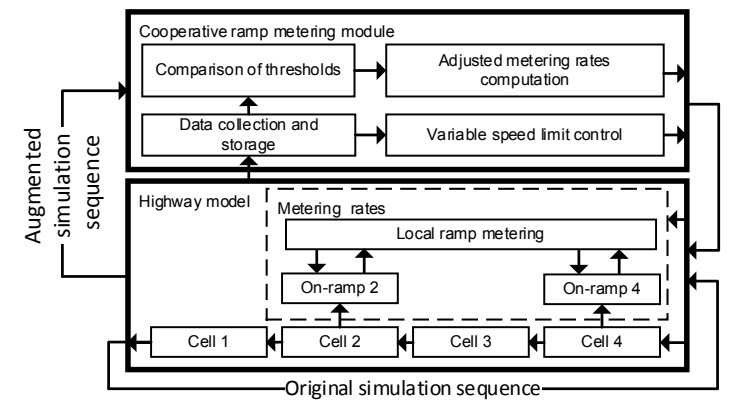

Figure 4. Augmented CTMSIM simulation structure

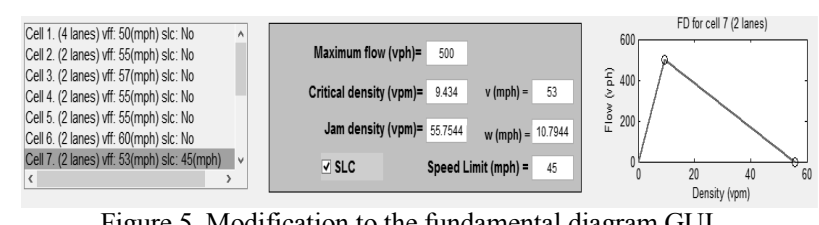

Figure 5. Modification to the fundamental diagram GUI to include the SLC option

computed by a local ramp metering algorithm. This feature is important for the HELPER algorithm which firstly computes metering rates for each on-ramp based on a local algorithm and then uses the cooperative module to obtain the final ramp metering value for each on-ramp.

\section{B. Speed limit module}

Second modification of CTMSIM involves implementation of SLC for every cell in the simulation model. SLC is implemented through modification of the cell mean speed equation given with (9) into:

$$
v_{i}^{c}=\min \left(v_{i}^{S L C}, \frac{f_{i[k]} /\left(1-\beta_{i[k]}\right)}{n_{i[k]}+\gamma r_{i[k]}}\left(\frac{L_{i}}{\Delta t}\right), v_{i}^{f f}\right),
$$

where $v_{i}^{S L C}$ denotes the current SLC value for $i$-th cell. SLC value must be lower than free flow speed value of the current cell. Original CTMSIM traffic fundamental diagram GUI is modified for definition of SLC parameters as presented in Fig. 5.

\section{SimULATION RESUlts}

To test the CTMSIM augmentations, different simulation scenarios were defined. Each traffic scenario includes increased traffic demand at the on-ramp in cell 6. Such scenario enables a simulation environment to test cooperative highway control strategies when congestion starts to build up. Obtained simulation results are divided into three groups. First group of simulation results contains comparative analysis of local ramp metering algorithms. Analysis is conducted using the following ramp metering algorithms: local (ALINEA), competitive (SWARM) and cooperative (HELPER). For comparison, simulation scenario without ramp metering control is also added to the analysis. Second group of simulation results are addressed to VSLC validation. Third simulation group presents cooperation of VSLC with the HELPER ramp metering algorithm.

\section{A. Zagreb bypass and traffic flow data}

Urban highway section between the nodes Jankomir and Lučko on the Zagreb bypass is selected to be the simulation use case model. Mentioned section contains 70 $\%$ traffic generated by the nearby town Zagreb [10]. Additionally, this section has increased traffic load during the whole day and also experiences a significant effect from daily migrations. Traffic demand for every on-ramp on this section is reconstructed according to Ljubljana bypass traffic flow data, since accurate hourly traffic flow data for the Zagreb bypass are not available. For this reconstruction Zagreb bypass average daily traffic flow from [10] and Ljubljana daily flow change characteristic were used. Graphical presentation of the ACTM model of highway section between nodes Jankomir and Lučko is presented in Fig. 6. Green colour represents on-ramps and blue colour off-ramps. First cell is denoted yellow. 


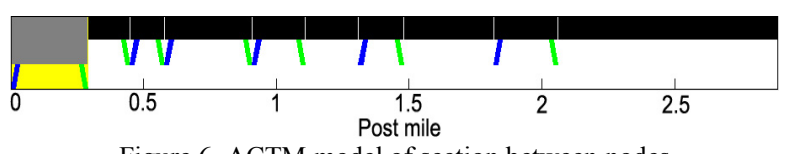

Figure 6. ACTM model of section between nodes Jankomir and Lučko

\section{B. Cooperation between on-ramps}

In order to compare obtained simulation results appropriate LoS measures have to be used. LoS measures used in this analysis are: travel time (TT) and delay. TT is a simple LoS measure which gives information how much time one vehicle needs to travel through observed highway segment. This measure is used to observe throughput of mainstream in the same time ignoring queues at on-ramps. TT is measured in minutes. Delay is defined as the difference between the actual time spent by all vehicles on a congested highway and the time spent in case they have travelled at free flow speed. Delay also considers vehicles which are waiting in on-ramp queues or in mainstream queues caused by the bottlenecks. It is measured in vehicle-hours.

Results of LoS analysis are shown in Tab. I. According to Tab. I. among the stand-alone ramp metering algorithms, cooperative algorithm HELPER has achieved best TT due to its restrictive nature. Lowest delay was achieved in the simulation scenario without ramp metering. In this basic scenario the blending coefficient $\gamma$ was set to 1 . This means that all vehicles from the on-ramps are immediately merged with mainstream under the condition that in a current cell maximal mainstream capacity is not exceeded. Such behaviour induces smaller on-ramps queues but significantly increases traffic density of mainstream which also increases average TT. These specific behaviours can be seen in Figs. 7 and 8.

HELPER maintains increased mainstream throughput by distributing vehicles and consequently the waiting time to the "slave" on-ramps queues. This behaviour causes longer queues at particular "slave" on-ramps increasing the delay, respectively. Additionally, "slave" on-ramps decrease the number of incoming vehicles from the upstream on-ramps regarding the current bottleneck location. This reduces impact of congestion backpropagation on the mainstream throughput which consequently decreases TT.

\section{Variable speed limit control}

Firstly, VSLC is analysed as a standalone highway traffic control strategy. Analysis includes comparison of simulation results obtained without and with applied VSLC at the same highway simulation model. VSLC is applied in the cells 2, 5 and 6. Main goal of VSLC is to

TABLE I. Results of cooperative analysis between different traffic control algorithms

\begin{tabular}{|c|c|c|}
\hline $\begin{array}{c}\text { Traffic control } \\
\text { algorithm }\end{array}$ & $\begin{array}{c}\text { TT } \\
\text { (min) }\end{array}$ & $\begin{array}{c}\text { Delay } \\
\text { (vehicle-hour) }\end{array}$ \\
\hline None & 7.06 & 15.87 \\
\hline ALINEA & 3.90 & 36.88 \\
\hline SWARM & 3.71 & 41.49 \\
\hline HELPER & 3.40 & 22.63 \\
\hline VSLC & 5.59 & 12.24 \\
\hline HELPER + VSLC & 3.30 & 21.50 \\
\hline
\end{tabular}

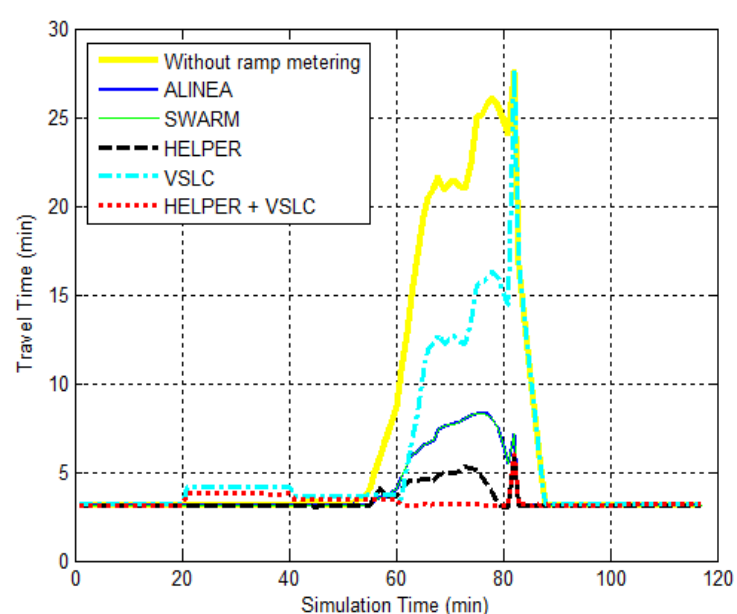

Figure 7. Results of comparative analysis according to travel time

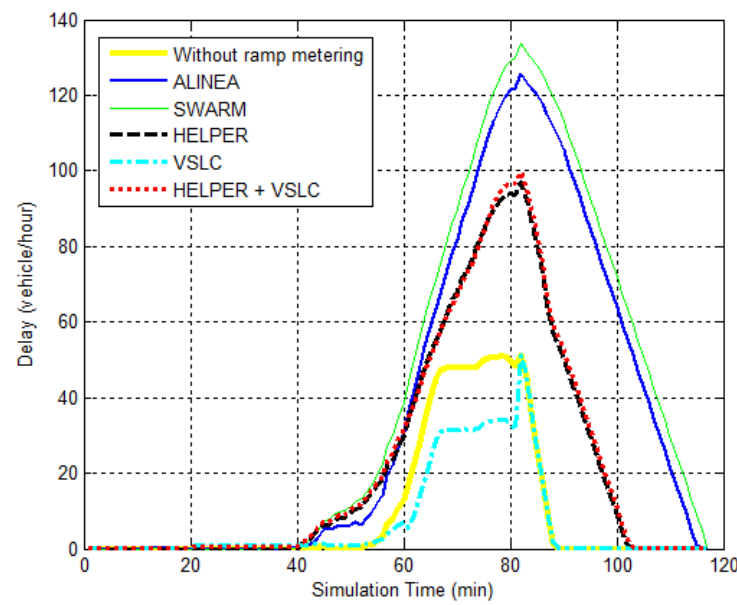

Figure 8. Results of comparative analysis according to delay

gradually decrease speed of the upstream flow before congestion starts. Such approach firstly gradually decreases mainstream speed, but enables higher mainstream speed during the congestion period unlike the scenario without VSLC.

Analysis of mainstream speeds can be seen in Fig. 9. VSLC prevented traffic standstill during most of the congested period. VSLC produces vehicle platoons which are traveling at lower speeds in comparison to the free flow speed by decreasing mainstream speed. This impacts the process of mainstream congestion back-propagation. From Fig. 10 it is possible to notice that mainstream density is lower at upstream cells of the congested cell 6
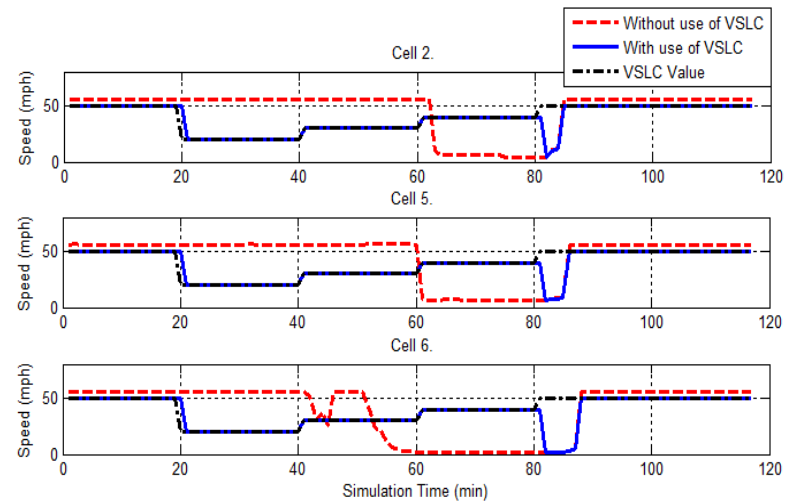

Figure 9. Analysis of mainstream speed with and without VSLC 

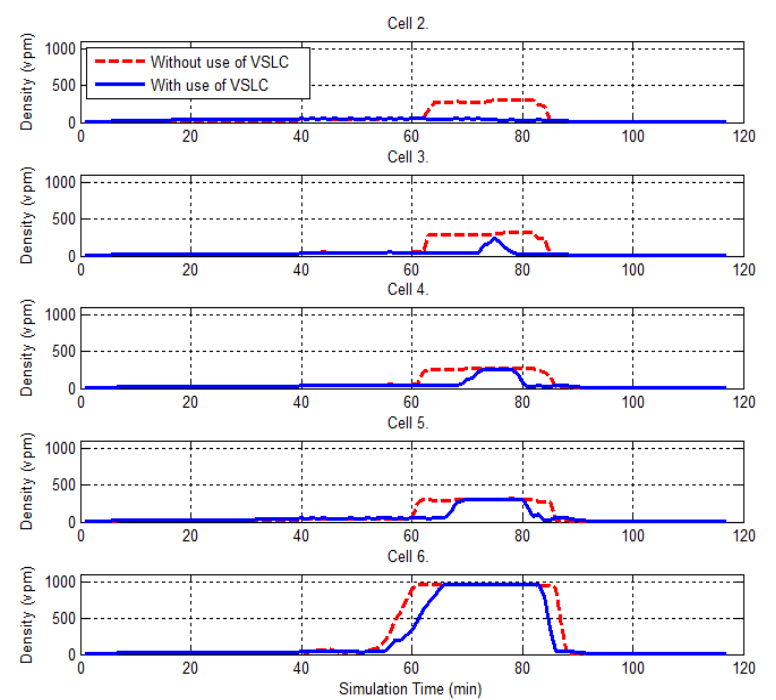

Figure 10. Analysis of mainstream density with and without the use of VSLC

with VSLC in contrast to the situation without VSLC.

\section{Cooperation between on-ramps and VSLC}

In this scenario cooperation between HELPER ramp metering algorithm and VSLC is simulated. Results of comparative analysis presented in Figs. 7 and 8 show that cooperation between HELPER ramp metering algorithm and VSLC produces smallest TT. VSLC decreases mainstream speed starting in the cell before the last "slave" on-ramp cell and ending in the congested cell. This decreases speed of the incoming vehicles to the area between last "slave" on-ramp and congested cell. Decreased speed of mainstream vehicles contributes to the lower values of density in the area between congested and last "slave" on-ramp. It can be concluded that virtual queues provided by cooperation between HELPER and VSLC significantly reduce traffic density upstream of the congested on-ramp. Lower upstream density of the congested on-ramp leaves additional mainstream capacity to accept vehicles which have origin from congestion back-propagation.

HELPER, as the standalone ramp metering algorithm and cooperation between HELPER and VSLC produce similar values of delay. The reason for such results is HELPER's reduction of metering rate on the "slave" onramps in order to reduce mainstream density upstream of the congested on-ramp. VSLC as the part of HELPER and VSLC cooperation does not have a strong influence on the delay as in the case of TT due to lower mainstream density. It can be concluded that VSLC has lower influence on delay if the mainstream density is decreased by HELPER exploitation of on-ramp queues. Standalone VSLC produces better delay results because of higher value of mainstream density. Other ramp metering algorithms produce higher values of delay due to higher number of vehicles in the congested on-ramp queue.

\section{CONCLUSION AND FUTURE WORK}

In this paper cooperative systems simulation in traffic control is tackled. Possibilities of cooperative control application in urban highway management are described. Cooperation between on-ramps in ramp metering, and between ramp metering and VSLC are chosen as most common systems. Simulation requirements are explained and framework for macroscopic highway traffic simulator CTMSIM augmentation is proposed. Proposed framework includes additional simulation step for cooperative control computations (between on-ramps and between ramp metering and VSLC) and augmentation of the ACTM highway model to include SLC. Both traffic control systems can be used as standalone or in cooperation.

To test implemented CTMSIM augmentations the ramp metering algorithm HELPER and VLSC are used. Simulations verify operational work of the proposed cooperative framework. All simulations are made using part of Zagreb bypass, the segment between nodes Lučko and Jankomir. Simulation results show that cooperation between VSLC and cooperative ramp metering provides best TT and delay values in comparison with other ramp metering algorithms involved into analysis.

Future work will include implementation of additional cooperative ramp metering algorithms and an optimization algorithm for cooperation between ramp metering and VSLC.

\section{ACKNOWLEDGMENT}

The research reported in this paper is partially funded by the FP7 - Collaborative Project: Intelligent Cooperative Sensing for Improved traffic efficiency - ICSI (FP7317671) and by the EU COST action TU1102. Authors wish to thank Nikola Bakarić for his valuable comments.

\section{REFERENCES}

[1] M. Treiber, and A. Kesting, "Traffic flow dynamics", SpringerVerlag Berlin Heidelberg, 2013.

[2] M. Gregurić, M. Buntić, E. Ivanjko, and S. Mandžuka, "Improvement of highway level of service using ramp metering", Proceedings of the $21^{\text {st }}$ international symposium on electronics in transport - ISEP 2013, Ljubljana, Slovenia, 25. - 26. March, 2013.

[3] D. Li, and P. Ranjitkar, "Assessing Ramp Metering and Variable Speed Limits Strategies for Auckland Motorway", ITS World Congress Tokyo, Japan, 2013, Paper 4161, 2013.

[4] M. C. Esposito, J. W. Polak, R. Krishnan, and M. Pleydell, "A Global Comparison of Ramp-Metering Algorithms Optimizing Traffic Distribution on Motorways and Arterials", Road Transport Information and Control Conference and the ITS United Kingdom Members' Conference, 25. - 27. May, 2010. pp. $1-6$.

[5] C. Lee, B. Hellinga, and K. Ozbayc, "Quantifying effects of ramp metering on freeway safety", Proceedings of $38^{\text {th }}$ Conference on Accident Analysis and Prevention, Waterloo, Ontario, Canada, 2006, pp. 279-288.

[6] M. Hasan, M. Jha, and M. Ben-Akiva, "Evaluation of ramp control algorithms using microscopic traffic simulation“, Transportation Research Part C 10, MIT, Cambridge, MA 02142, USA, 2010. pp. 229-256.

[7] M. Papageorgiou, C. Diakaki, V. Dinopoulou, Y. Wangand, and A. Kotsialos, "Review of Road Traffic Control Strategies", Proceedings of the IEEE, Vol. 91, No. 12, December, 2003, pp 2043-2067.

[8] A. Kurzhanskiy, and P. Varaiya, "CTMSIM Traffic MacroSimulator for MATLAB", User Guide, TOPL Group, UC Berkeley, 2008.

[9] G. Gomes, and R. Horowitz, "Optimal freeway ramp metering using the asymmetric cell transmission model", Transportation Research Part C 14, 2006. pp. 244-262.

[10] G. Štefančić, D. Marijan, and S. Kljajić, "Capacity and Level of Service on the Zagreb Bypass", Promet - Traffic\&Transportation, Zagreb, Croatia, Vol. 24, No. 3, 2012. pp. 261-267 